\title{
Seuil des D-dimères ajusté à l'âge chez les patients de plus de 75 ans : une étude rétrospective.
}

Age dependent D-dimer cut-off for the elderly patient in the emergency room.

Paquet AL,1, Balia D,1, Dubreucq E,1, Roger C,1, Hausfater P,1,2, Freund Y,1,2.

1) Service d'accueil des urgences, Groupe hospitalier Pitié Salpétrière, Assistance publique hôpitaux de Paris, Paris, France

2) Paris Sorbonne universités, UPMC Univ Paris 06, Paris, France

Auteur correspondant :

Anne Laure Paquet,

Service d'Accueil des Urgences, CHU Pitié-Salpêtrière,

47-83 boulevard de l'hôpital, 75651 Paris Cedex 13.

annelaure.paquet@gmail.com

06.72.07.18.86

Mots clés : Embolie pulmonaire - D-dimères - Urgences 


\section{Résumé :}

Introduction: Devant les limites de la stratégie diagnostique classique de l'embolie pulmonaire (EP) chez le patient âgé, l'intérêt d'un seuil de D-dimères adapté à l'âge (10xâge) a été récemment validé. Notre étude a pour but d'évaluer l'utilisation de ces nouvelles recommandations au sein de notre service d'urgence.

Matériels et méthodes : Nous avons réalisé une étude rétrospective, sur l'année 2015, dans un service d'urgence urbain universitaire. Tous les patients de plus de 75 ans ayant subi un dosage de D-dimères pour suspicion d'EP au cours de l'année 2015 ont été inclus. L'objectif principal de l'étude était d'estimer l'adhérence aux recommandations sur le seuil ajusté de Ddimères.

Résultats : Cent vingt-six patients ont été inclus, dont 79 (63\%) femmes. Trente patients (24\% de la population) avaient un résultat de D-dimères inférieur au seuil ajusté à l'âge mais supérieur à $500 \mu \mathrm{g} / \mathrm{l}$. Parmi eux, quatre (13\%) ont eu une angio-tomodensitométrie thoracique (AngioTDM), soit un taux d'adhérence aux recommandations de 87\% (95\% CI 70\% - 97\%). Aucune EP n'a été retrouvée chez ces quatre patients.

Conclusion: Les recommandations pour l'utilisation du seuil de D-dimères adapté à l'âge chez les patients de plus de 75 ans dans les suspicions d'embolie pulmonaire ne sont pas parfaitement respectées dans notre service d'urgence.

Mots clés : Embolie pulmonaire - D-dimères - Patient âgé - Seuil ajusté - Angio-TDM thoracique 


\begin{abstract}
:
Aims: The pulmonary embolism (PE) incidence is increased in elderly patient. The conventional strategy to diagnose PE in elderly population is limited by the low specificity of d-dimer testing, and the interest of an age-adjusted D-dimer cut-off (10xage) was recently validated. The aim of this study was to evaluate the use of these new recommendations in our emergency department. Procedure: We performed a retrospective study on all emergency patients aged 75 and older who were tested for PE in 2015 in our hospital. The primary objective of this study was to assess the use of the recommendation for age adjusted cut-off. Results: One hundred and twenty six patients were enrolled. Thirty patients $(24 \%)$ had negative D-dimer considering the age adjusted cut-off but higher than the usual threshold of $500 \mu \mathrm{g} / \mathrm{l}$. In this group, four (13\%) underwent a CT pulmonary angiogram, which means that emergency physicians applied the new guidelines in $87 \%$ cases (95\% CI 70\% - 97\%). No PE was found among these four patients. Conclusion: The age-adjusted D-dimer cut-off in elderly patients with suspicion of PE doesn't seems to be well endorsed in our emergency department.
\end{abstract}

Keywords: Pulmonary embolism - D-dimer - Elderly patient - Age adjusted cut-off - CT scanner 


\section{Introduction :}

L'embolie pulmonaire (EP) représente la troisième cause de mortalité cardiovasculaire [1].

Son incidence en France est estimée à plus de 60 cas pour 100000 par an avec une probable sous-estimation de ce chiffre [2,3]. L'incidence de la thrombose veineuse profonde est 10 fois supérieure après 70 ans [4]. La mortalité après un évènement thromboembolique est plus élevée chez le patient de plus de 65 ans, avec un taux de décès de $31 \%$ à un an, contre $14 \%$ chez les moins de 65 ans [5]. La présentation clinique de l'EP chez le patient âgé peut être frustre et les signes cardinaux tels que la dyspnée ou la douleur thoracique peuvent être plus fréquemment attribués à d'autres pathologies que chez le sujet jeune. De plus, les troubles cognitifs (aigus ou chroniques) peuvent compliquer la recherche des signes typiques [6]. Son diagnostic peut être difficile pour le médecin urgentiste avec d'une part un risque d'examens complémentaires inutiles, et d'autre part la méconnaissance de cette pathologie lors d'une présentation atypique non grave. Les stratégies diagnostiques reposent sur l'utilisation séquentielle de 3 points: 1) Evaluation de la probabilité clinique d'EP faible, intermédiaire ou forte (score de Wells ou de Genève ou probabilité clinique empirique «gestalt »);2) Dosage des D-dimères : avec un seuil généralement fixé à $500 \mu \mathrm{g} / 1$; 3) Réalisation d'une imagerie thoracique injectée $[1,7,8]$.

La spécificité des D-dimères pour le diagnostic d'EP diminue avec l'âge car leur concentration plasmatique moyenne augmente avec l'âge [9]. Le seuil de $500 \mu \mathrm{g} / \mathrm{l}$ permet d'exclure $60 \%$ des EP chez les moins de 40 ans mais seulement $5 \%$ après 80 ans $[10,11]$. L'évaluation d'un seuil adapté à l'âge a été réalisée pour la première fois en 2010 par une étude rétrospective de 4 cohortes prospectives [1,12]. En 2013 une méta-analyse de 13 cohortes a confirmé une augmentation de la spécificité du test avec le seuil ajusté à l'âge, sans perte significative sur le taux de faux négatifs [13]. Ces résultats ont été confirmés par une étude multicentrique conduite entre 2010 et 2013 [14]. Ce seuil, D-dimères = âge x 10 au-delà de 50 ans, fait partie de la stratégie diagnostique de l'EP dans les nouvelles recommandations de l'ESC 2014 [1]. Notre étude a pour but d'évaluer l'utilisation de ces nouvelles recommandations au sein de notre service d'urgence. 


\section{Méthode :}

Nous avons réalisé une étude rétrospective monocentrique sur l'année 2015, dans un service d'urgences urbain universitaire accueillant près de 70000 consultations par an. En tant qu'étude rétrospective non interventionnelle, le Comité de Protection des Personnes de Paris VI - Ile de France, n'a pas requis de consentement des patients.

Nous avons récupéré, via le logiciel informatique Urqual ${ }^{\circledR}$, les dossiers informatisés des patients de 75 ans et plus ayant eu un dosage de D-dimères aux urgences entre le $1^{\text {er }}$ janvier 2015 et le 31 décembre 2015. Ces patients se présentaient avec une suspicion d'EP, la probabilité clinique faible ou intermédiaire d'EP n'a pas été retrouvée dans les dossiers médicaux étudiés. Les suspicions isolées de thrombose veineuse profonde du membre inférieur ont été exclues. Les données relatives aux patients, les valeurs de D-dimères, la réalisation ou non d'une imagerie et ses résultats ont été récupérés et analysés. La technique de dosage des D-dimères utilisé était la technique EFLA (VIDAS) recommandée dans l'étude ADJUST [14].

L'objectif principal de l'étude était d'estimer l'adhérence aux recommandations sur le seuil ajusté de D-dimères, en déterminant la fréquence d'écart à ce protocole pour les patients de plus de 75 ans présentant une suspicion d'EP aux urgences. Le critère de jugement principal était la réalisation d'une angioTDM malgré des D-dimères inférieur au seuil ajusté à l'âge mais supérieur à $500 \mu \mathrm{g} / \mathrm{l}$, soit le taux de suivi des nouvelles recommandations parmi les patients avec des D-dimères supérieur à $500 \mu \mathrm{g} / \mathrm{l}$ mais inférieur au seuil de 10xâge. Les critères de jugement secondaires incluaient la réalisation d'une angioTDM malgré des Ddimères inférieur au seuil ajusté à l'âge (y compris $<500 \mu \mathrm{g} / \mathrm{l}$ ), et le taux de positivité des angioTDM réalisées hors recommandation.

\section{Analyse statistique :}

Les variables qualitatives étaient exprimées en effectifs (pourcentage \%) et l'intervalle de confiance exact à 95\% (IC 95\%) était calculé. Les variables quantitatives en moyenne (déviation standard DS) ou en médiane (interquartile range IQR) selon leur distribution normale ou non. Les analyses ont été effectuées à l'aide du logiciel NCSS (Statistical Solution, Cork Ireland, V11).

\section{Résultats :}

Nous avons inclus 126 patients de 75 ans ou plus ayant bénéficié d'un dosage de D-dimères pour suspicion d'EP aux urgences sur l'année 2015. Cette population était majoritairement 
féminine avec 79 femmes (63\%) et 47 hommes (37\%). L'âge médian était de 82 ans (IQR, 79-86). Le motif de consultation était pour 56 (44\%) patients une dyspnée ou une toux et pour $32(25 \%)$ d'entre eux une douleur thoracique.

Sur l'ensemble de la cohorte, 52 (41\%) patients avaient un taux de D-dimères négatif selon les nouvelles recommandations : 22 patients avaient des D-dimères $<500 \mu \mathrm{g} / 1$ et 30 patients des D-dimères $<$ âgex 10 mais $\geq 500 \mu \mathrm{g} / 1$ (fig. 1). Huit EP ont été diagnostiquées dans notre échantillon, soit une prévalence de 6\% (IC95\%, 3\% - 12\%).

Sur les 30 patients ayant des D-dimères $\geq 500 \mu \mathrm{g} / 1$ mais inférieur au seuil de 10xâge, quatre (13\%) patients ont eu une angioTDM, soit un taux de suivi des recommandations à $87 \%(95 \%$ CI 70\% - 97\%). Après relecture des quatre dossiers, trois angioTDM étaient évitables. La recherche d'une dissection aortique pour la quatrième patiente est cohérente.

Quatre autres patients avec des D-dimères $<500 \mu \mathrm{g} / 1$ ont bénéficié d'une angioTDM thoracique dans le cadre du bilan de leur dyspnée. Deux d'entre eux présentaient une hémoptysie, le médecin recherchait une étiologie ou d'un saignement actif à l'aide d'une angioTDM. Pour les deux autres patients l'angioTDM a été réalisée comme bilan d'une dyspnée et les diagnostics étaient respectivement une pneumopathie virale et une décompensation de BPCO.

$\mathrm{Au}$ total, une angioTDM était demandée par excès pour huit (15\%) patients dans le groupe des D-dimères négatifs. Aucun de ces scanners ne révélait d'EP.

Soixante quatorze patients avaient des D-dimères positifs ( $\geq$ âgex10), 44 angioTDM ont été réalisées et une EP a été confirmée chez huit d'entre eux (soit 11\% des patients avec des Ddimères au dessus du seuil). Les diagnostics alternatifs prépondérants étaient un OAP pour 8 (33\%) patients et une pneumopathie hypoxémiante pour $2(8 \%)$ patients. Trente $(40 \%)$ de ces patients avec des D-dimères positifs n'ont pas bénéficié d'une angioTDM.

\section{Discussion :}

Dans cette étude rétrospective, on note que les nouvelles recommandations sur le seuil ajusté à l'âge des D-dimères ne semblent pas totalement intégrées, et qu'une proportion non négligeable de patients âgés a bénéficié d'une angioTDM sans indication. Au total, quatre patients sur 126 auraient pu voir leur suspicion d'EP écartée sur le résultat de D-dimères, mais ont dû passer une angioTDM malgré tout, soit un taux global de 3\% (13\% si on considère uniquement la population d'intérêt avec des valeurs de D-dimères intermédiaires entre 500 $\mu \mathrm{g} / 1$ et le seuil ajusté à l'âge). 
Trente (40\%) patients ayant eu un dosage de D-dimères positifs n'ont pas bénéficiés d'une angioTDM. Six d'entre eux avaient une contre-indication à l'injection d'iode (insuffisance rénale aigue, surcharge par OAP...). Ils ont tous été traités par anticoagulation efficace. En effet la population des plus de 75 ans ayant un taux d'insuffisance rénale plus élevé que la population générale, l'indication à une imagerie avec injection de produit de contraste s'en trouve limitée. Un autre diagnostique positif avait été retrouvé chez les 24 autres patients. Les diagnostics prépondérants étaient l'OAP pour $8(33 \%)$ patients et une pneumopathie hypoxémiante pour $2(8 \%)$ patients. On pourrait donc s'interroger sur l'indication de la prescription initiale du dosage des D-dimères chez ces 24 patients. Cependant la présentation de l'EP chez le patient âgé peut être atypique. L'EP dans cette population est généralement un diagnostic différentiel à d'autres affections cardio-pulmonaire. Seule l'association des examens complémentaires et de la clinique permet de poser un diagnostic final. Le caractère rétrospectif de notre étude ne nous à pas permis de connaître le devenir de ces patients. L'ajustement du seuil de D-dimères = âgex 10 chez le patient de plus de 50 ans permet d'augmenter la spécificité du test sans diminuer sa sensibilité, et surtout de réduire le recours aux examens d'imagerie grâce à l'exclusion du diagnostic d'EP chez 30\% des patients de plus de 75 ans (contre 6,4\% avec la valeur seuil standard de $500 \mu \mathrm{g} / 1$ ) [14]. Dans notre étude la proportion de patients de plus de 75 ans avec des D-dimères négatifs passe de $17 \%$ avec le seuil de $500 \mu \mathrm{g} / 1$ à $41 \%$ avec le seuil adapté à l'âge ce qui signifie que l'EP peut être exclue avec le seul dosage des D-dimères chez presque un patient sur deux. Ces résultats suggèrent une meilleure prise en charge des patients de plus de 75 ans en confirmant les nouvelles indications d'angioTDM. Cela permet une prise en charge plus rapide et une diminution du nombre d'examens complémentaires.

Notre recueil de données sur l'année 2015 confirme la bonne utilisation du seuil ajusté à l'âge chez les patients de plus de 75 ans. Quatre vingt sept pourcent des patients ont eu une prise en charge conforme aux recommandations sur les D-dimères ajustés à l'âge. Cependant il ne faut pas oublier la stratégie diagnostique avec l'évaluation du risque d'embolie pulmonaire et utiliser le dosage des D-dimères seulement dans les cas de suspicion faible ou intermédiaire d'EP. Notre étude ne permettait pas d'avoir accès au résultat du calcul éventuel d'une probabilité clinique pré-test et donc de valider la bonne indication du dosage des $\mathrm{D}$-dimères. Un total de $30(24 \%)$ patients avaient des D-dimères $\geq 500 \mu \mathrm{g} / 1$ mais inférieur au seuil de 10xâge. Cette proportion est comparable avec celle rapportée dans plusieurs études dont celle de Righini et al. [14]. Au total la réalisation d'une angioTDM aurait pu être évitée chez huit patients $(15 \%)$ si les recommandations avaient été strictement respectées. L'utilisation de 
l'angioTDM thoracique pour rechercher un diagnostic différentiel de l'EP n'est pas recommandée [15]. On peut s'interroger sur l'intérêt initial du dosage des D-dimères chez 5 $(10 \%)$ de ces patients pour qui l'on réalisait un bilan de dyspnée ou d'hémoptysie. Cependant la décision de prescription appartient au final au clinicien même si cette décision ne fait pas partie de l'algorithme habituel. L'interprétation du résultat est ainsi fonction de l'indication initiale et des autres résultats complémentaires.

En l'absence d'ajustement des D-dimères à l'âge, la réalisation de 104 scanners aurait été indiquée alors qu'en utilisant le seuil ajusté à l'âge seulement 74 scanners auraient dû être réalisés. On note donc une réduction de 30 (24\%) scanners.

Ces résultats semblent par ailleurs confirmer l'utilité et la sécurité diagnostique de l'utilisation d'un seuil adapté à l'âge afin d'éviter la réalisation d'imageries inutiles et potentiellement délétères pour les patients âgés : aucune EP n'a été retrouvé chez les patients à D-dimères négatifs. Ces résultats concordent avec ceux des précédentes études similaires [12,16]. Une des limites de cette étude est le fait que, n'ayant pas fait de scanner à tous les patients ayant des D-dimères inférieurs au seuil adapté à l'âge, on ne peut pas affirmer qu'ils n'avaient pas d'EP. Le caractère rétrospectif de notre étude ne permettait pas de faire un suivi au long cours de ses patients. Une autre limite provient du fait que la probabilité pré-test ne peut être clairement établie à la lecture du compte-rendu, et certain patients pouvaient être classés à haut risque et donc nécessiter une angioTDM quel qu'ait été le résultat de D-dimères. Par ailleurs, n'ayant pas fixé à priori de taux d'écart aux recommandations « acceptable », il est difficile de conclure et d'interpréter notre résultat principal.

Enfin, la prévalence d'EP dans notre étude est de 6\%, valeur relativement basse par rapport aux études européennes mais qui reflète probablement la pratique clinique réelle.

\section{Conclusion :}

L'ajustement du seuil de D-dimères en fonction de l'âge chez les patients de plus de 75 ans n'est pas parfaitement respecté dans notre échantillon. Un taux substantiel de patients se voit prescrire une imagerie thoracique en dehors des recommandations. Ces recommandations doivent être rappelées aux médecins pour limiter le nombre d'examens irradiants inutiles lors des différentes réunions médicales de service. Une nouvelle étude prospective après rappel des recommandations permettrait d'évaluer l'impact des réunions d'information sur l'adéquation des pratiques par rapport aux recommandations.

Liens d'intérêts : Les auteurs déclarent ne pas avoir de lien d'intérêt. 


\section{Références:}

1. Members AF, Konstantinides S, Torbicki A, et al (2014) 2014 ESC Guidelines on the diagnosis and management of acute pulmonary embolism. Eur Heart J 35:3033-73

2. Oger E (2000) Incidence of venous thromboembolism: a community-based study in Western France. EPI-GETBP Study Group. Groupe d'Etude de la Thrombose de Bretagne Occidentale. Thromb Haemost 83:657-60

3. Bouée S, Emery C, Samson A, et al (2016) Incidence of venous thromboembolism in France: a retrospective analysis of a national insurance claims database. Thromb J 14:4

4. Jeandel C (2006) Epidémiologie et facteurs de risque de la maladie thrombo-embolique veineuse du sujet âgé. Sang Thromb Vaiss 18:226

5. Spencer FA, Gore JM, Lessard D, et al (2008) Venous thromboembolism in the elderly. A community-based perspective. Thromb Haemost 100:780-8

6. Masotti L, Ceccarelli E, Forconi S, et al (2003) Diagnostic and therapeutic features of pulmonary embolism in elderly patients. Ital Heart J Suppl Off J Ital Fed Cardiol 4:74554

7. Becattini C, Agnelli G, Lankeit M, et al (2016) Acute pulmonary embolism: mortality prediction by the 2014 European Society of Cardiology risk stratification model. Eur Respir J 48:780-86

8. Ray P, Le Manach Y, Riou B, et al (2010) Statistical evaluation of a biomarker. Anesthesiology 112:1023-40

9. Masotti L, Ceccarelli E, Cappelli R, et al (2000) Plasma D-dimer levels in elderly patients with suspected pulmonary embolism. Thromb Res 98:577-9

10. Righini M, Goehring C, Bounameaux H, et al (2000) Effects of age on the performance of common diagnostic tests for pulmonary embolism. Am J Med 109:357-61

11. Righini M, Le Gal G, Perrier A, et al (2005) The challenge of diagnosing pulmonary embolism in elderly patients: influence of age on commonly used diagnostic tests and strategies. J Am Geriatr Soc 53:1039-45

12. Douma RA, le Gal G, Söhne M, et al (2010) Potential of an age adjusted D-dimer cut-off value to improve the exclusion of pulmonary embolism in older patients: a retrospective analysis of three large cohorts. BMJ 340:1475

13. Schouten HJ, Geersing GJ, Koek HL, et al (2013) Diagnostic accuracy of conventional or age adjusted D-dimer cut-off values in older patients with suspected venous thromboembolism: systematic review and meta-analysis. BMJ 346:2492

14. Righini M, Van Es J, Den Exter PL, et al (2014) Age-adjusted D-dimer cutoff levels to rule out pulmonary embolism: the ADJUST-PE study. JAMA 311:1117-1124

15. Chandra S, Sarkar PK, Chandra D, et al (2013) Finding an alternative diagnosis does not justify increased use of CT-pulmonary angiography. BMC Pulm Med 13:9 
16. Flores J, García de Tena J, Galipienzo J, et al (2016) Clinical usefulness and safety of an age-adjusted D-dimer cutoff levels to exclude pulmonary embolism: a retrospective analysis. Intern Emerg Med 11:69-75 


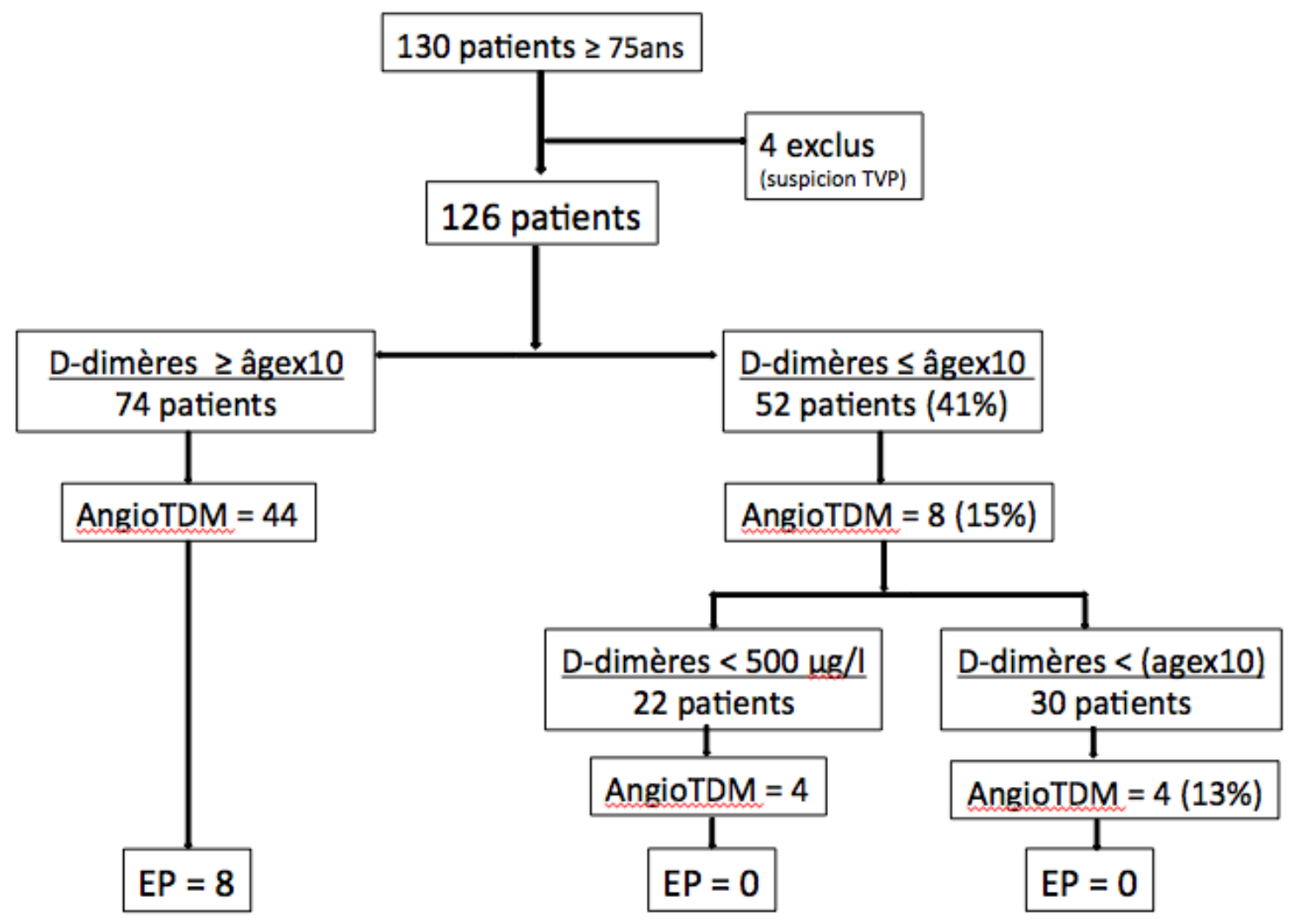

Fig. 1 Diagramme de flux (TVP: thrombose veineuse profonde. AngioTDM: Angio Tomodensitométrie) 